\title{
THE SPACE OF EXPONENTIALLY DECREASING ENTIRE FUNCTIONS AND ITS APPLICATION TO SOLVABILITY
}

\author{
DOHAN KIM \\ Seoul National University, Seoul 151-742, Korea \\ SOON-YEONG CHUNG \\ Duksung Women's University, Seoul 132-714, Korea
}

Introduction. It is well known that the Mizohata equation

$$
\frac{\partial u}{\partial t}+i t \frac{\partial u}{\partial x}=f, \quad f \in C_{0}^{\infty}\left(\mathbb{R}^{2}\right),
$$

has no solution in the space $\mathcal{D}^{\prime}\left(\mathbb{R}^{2}\right)$ of distributions, or in the space $B\left(\mathbb{R}^{2}\right)$ of hyperfunctions (see $[\mathrm{T}]$ for the historical backgrounds). On the other hand, N. Aronszajn introduced an abstract Fréchet space, the Aronszajn space of the traces of the analytic solutions of the heat equations in $\mathbb{C}^{n} \times\left\{t \in \mathbb{C}^{1}\right.$, Ret $\left.>0\right\}$, and M. S. Baouendi $[B]$ simplified the complicated arguments and showed that this equation has a solution in the Aronszajn space.

In this talk, we introduce a much simpler space $\mathcal{X}\left(\mathbb{R}^{n}\right)$ of real analytic and exponentially decreasing functions and show that the Fourier transformation is an isomorphism on $\mathcal{X}\left(\mathbb{R}^{n}\right)$, and also on its strong dual $\mathcal{X}^{\prime}\left(\mathbb{R}^{n}\right)$. As an application, applying the Fourier transformation only we show that the Mizohata operator is solvable in $C^{\infty}\left(\mathbb{R}_{t} ; \mathcal{X}^{\prime}(\mathbb{R})\right)$.

The complete proofs will be published elsewhere.

1. Definitions and basic properties. We introduce a space of exponentially decreasing functions and its strong dual.

Definition 1.1. We denote by $\mathcal{X}$ or $\mathcal{X}\left(\mathbb{R}^{n}\right)$ the set of all $\phi \in C^{\infty}\left(\mathbb{R}^{n}\right)$ such

This work was partially supported by the GARC-KOSEF. 
that for any $k, h>0$

$$
|\phi|_{k, h}=\sup _{\substack{x \in \mathbb{R}^{n} \\ \alpha \in \mathbb{N}_{0}^{n}}} \frac{\left|\partial^{\alpha} \phi(x)\right| \exp k|x|}{h^{|\alpha|} \alpha !}<\infty
$$

where $\mathbb{N}_{0}$ is the set of all nonnegative integers. The topology in $\mathcal{X}$ defined by the semi-norms in (1.1) makes $\mathcal{X}$ a Fréchet space. In fact, it is the projective limit topology over all $h>0$ and $k>0$.

Furthermore, the space $\mathcal{X}$ is a Fréchet nuclear space and therefore it is reflexive. Also, it is easy to show that the space $\mathcal{X}\left(\mathbb{R}^{n}\right)$ is dense in $\mathcal{S}$.

We show the Fourier transformation is an isomorphism of $\mathcal{X}\left(\mathbb{R}^{n}\right)$.

THEOREM 1.2. The Fourier transformation $\mathcal{F}: \phi \rightarrow \widehat{\phi}$ is a topological isomorphism of $\mathcal{X}\left(\mathbb{R}^{n}\right)$ with inverse given by the Fourier inversion formula.

Definition 1.3. We denote by $\mathcal{X}^{\prime}$ the strong dual of $\mathcal{X}$. In other words, $u \in \mathcal{X}^{\prime}$ if and only if there exist $k, h>0$ and $C=C(k, h)>0$ such that

$$
|u(\phi)| \leq C|\phi|_{k, h}, \quad \varphi \in \mathcal{X}
$$

It is clear that the space $\mathcal{S}^{\prime}$ of tempered distributions is a subclass of $\mathcal{X}^{\prime}$ by Theorem 1.2. Finally, we have the following theorem.

THEOREM 1.4. The Fourier transformation is an isomorphism of $\mathcal{X}^{\prime}\left(\mathbb{R}^{n}\right)$.

2. Applications. We are in a position to state the main result of our talk.

THEOREM 2.1. The Mizohata equation

$$
\frac{\partial u}{\partial t}+i t^{k} \frac{\partial u}{\partial x}=f, \quad f \in C_{0}^{\infty}\left(\mathbb{R}^{2}\right),
$$

has a solution in the space $C^{\infty}\left(\mathbb{R}_{t} ; \mathcal{X}^{\prime}(\mathbb{R})\right)$.

Finally, we show that the space $\mathcal{X}\left(\mathbb{R}^{n}\right)$ is stable under local operators.

TheOREM 2.2. Let $P(x, D)=\sum_{|\alpha|=0}^{\infty} a_{\alpha}(x) D^{\alpha}$ be a local operator, i.e., the differential operator of infinite order with the property that for any $M>0$ there exist $L>0$ and $B>0$ such that

$$
\sup _{x \in \mathbb{R}^{n}}\left|D^{\beta} a_{\alpha}(x)\right| \leq B M^{|\beta|} \beta ! L^{|\alpha|} / \alpha !
$$

for all $\alpha$ and $\beta$. Then the operator $P(x, D): \mathcal{X} \rightarrow \mathcal{X}$ is continuous.

\section{References}

[B] M. S. Baouendi, Solvability of partial differential equations in the traces of analytic solution of the heat equation, Amer. J. Math. 97 (1976), 983-1005.

[K] H. Komatsu, Ultradistributions, I. Structure theorems and a characterization, J. Fac. Sci. Univ. Tokyo Sect. IA Math. 20 (1973), 25-105. 
[M] T. Matsuzawa, A calculus approach to hyperfunctions. II, Trans. Amer. Math. Soc. 313 (1989), 619-654.

[PM] Y. Park and M. Morimoto, Fourier ultra-hyperfunctions in the Euclidean n-space, J. Fac. Sci. Univ. Tokyo Sect. IA Math. 20 (1973), 121-127.

[S] P. Schapira, Une équation aux dérivées partielles sans solutions dans l'espace des hyperfonctions, C. R. Acad. Sci. Paris Sér. A 265 (1967), 665-667.

[T] F. Treves, On local solvability of linear partial differential equations, Bull. Amer. Math. Soc. 76 (1970), 552-571. 